\title{
Hyperbolic Raisa Orbits of the Second Order in an Extended Hyperbolic Plane
}

\author{
Lyudmila N. Romakina* \\ (Dedicated to the memory of Prof. Dr. Aurel BEJANCU (1946 - 2020))
}

\begin{abstract}
In this paper, we study conics, which are invariant under the hyperbolic inversion with respect to the absolute of an extended hyperbolic plane $H^{2}$ of curvature radius $\rho, \rho \in \mathbb{R}_{+}$. They are called the hyperbolic Raisa Orbits of the second order. We prove that each hyperbolic Raisa Orbits of the second order in $H^{2}$ belongs to one of four conics types of this plane. These types are as follows: the bihyperbolas of one sheet; the hyperbolas; the hyperbolic parabolas of one sheet and two branches; the elliptic cycles of radius $\pi \rho / 4$. The family of all hyperbolic Raisa Orbits from the family of all bihyperbolas of one sheet (or all hyperbolas) defined exactly up to motions, is one-parametric. The family of all hyperbolic Raisa Orbits from the family of all hyperbolic parabolas of one sheet and two branches (or all elliptic cycles) contains a unique conic defined exactly up to motions.
\end{abstract}

\section{Introduction}

\subsection{The extended hyperbolic plane $H^{2}$}

In the Cayley-Klein projective interpretation (see, for instance, $[4,10,9])$, an extended hyperbolic plane $H^{2}$ is the projective plane $P_{2}$ with an infinitely removed oval curve $\gamma$, called the absolute of the plane $H^{2}$. According to Staudt's definition, the oval curve is also named a non-degenerate conic or simply a conic (see, for instance, [1]). The plane $H^{2}$ contains two connected components adjacent to the absolute. A complete Lobachevskii plane $\Lambda_{2}$ is realized on the interior domain of the plane $P_{2}$ with respect to the conic $\gamma$. A hyperbolic plane $\widehat{H}$ of positive curvature is realized on the exterior domain of $P_{2}$ with respect to $\gamma[13,14]$. The group $G$ of projective automorphisms of the oval curve $\gamma$ is the fundamental group of transformations for $H^{2}, \Lambda_{2}$, and $\widehat{H}$.

The lines of the plane $H^{2}$ are as follows:

(1) the elliptic lines, crossing the absolute at a pair of imaginary conjugate points;

(2) the hyperbolic lines, intersecting the absolute at two real points;

(3) the parabolic lines that touch the absolute.

\subsection{The conics types of the extended hyperbolic plane $H^{2}$}

Each conic of the plane $H^{2}$, different from the absolute, belongs to one of twenty types. Each proper conic of the Lobachevskii plane $\Lambda_{2}$ belongs to one of five types [9, p. 181], and each proper conic of the plane $\widehat{H}$ belongs to one of eight types [14,11]. Notice, the refined conics classification in the plane $\Lambda_{2}$ was provided in [9]. One of the conics types, namely the hyperbolic parabolas of one branches in $\Lambda_{2}$, was skipped in [5], [4, p. 229].

The classification of conics in the planes $\Lambda_{2}$ and $\widehat{H}$ (see $[4,10,14,11,5]$ ) is based on the classification of collineations, which are the product of polar transformations of two conics. In this classification, the geometric covariants of two conics of the projective plane $P_{2}$ are considered, namely the common points and the common 
tangents of a conic and the absolute. These geometric covariants are called the basic covariants of a conic [11]. Each a basic covariant can be real or imaginary. In the conics classification, the number of basic covariants and their nature are taken into account. Other ways for the conics classification are known. For example, focaldirector properties of conics were used in the classification of conics of the plane $\Lambda_{2}[7,8]$. Interestingly, the relative position of two projective conics is also investigated regardless of hyperbolic geometry, without taking into account the classic results from the works $[4,10,5]$. For example, incomplete lists of the possible relative positions of conics are presented in [6,3]. Such investigations, despite their omissions, confirm the timeliness of the study of the conics in the plane $H^{2}$. In the given paper, we adhere to the conics classification from $[10,14,11]$ and use terms from the works [14,11]. All types of conics of the plane $H^{2}$ are shown in Figure 1.

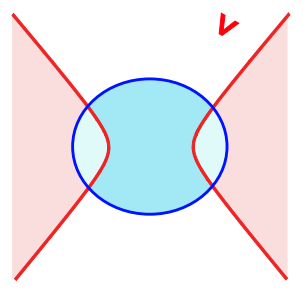

a
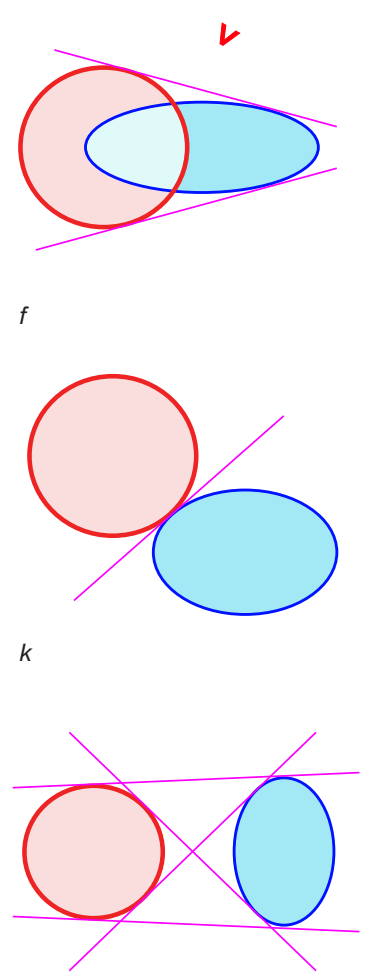

$p$
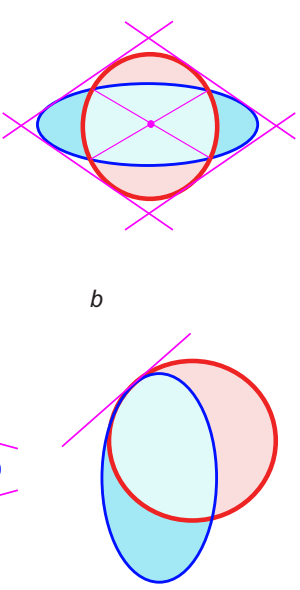

$g$

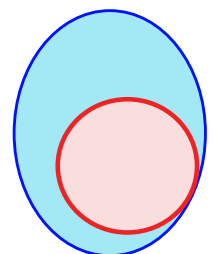

1

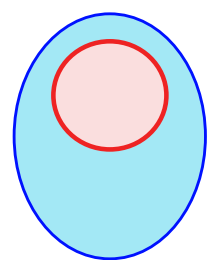

$q$

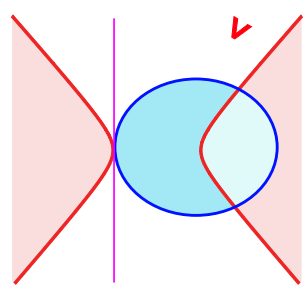

c

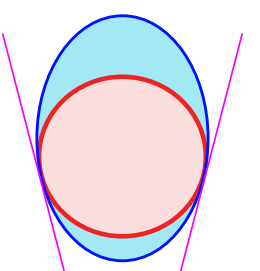

h

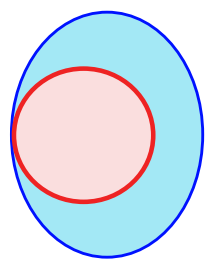

$m$

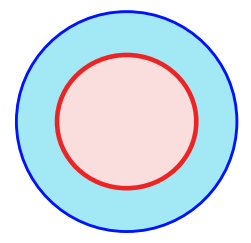

$r$
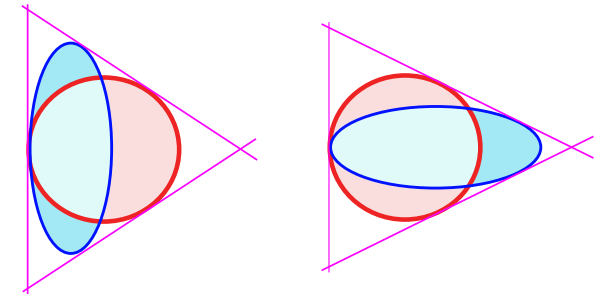

d

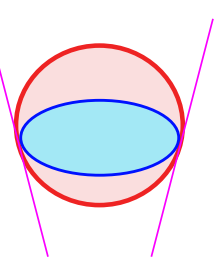

$i$
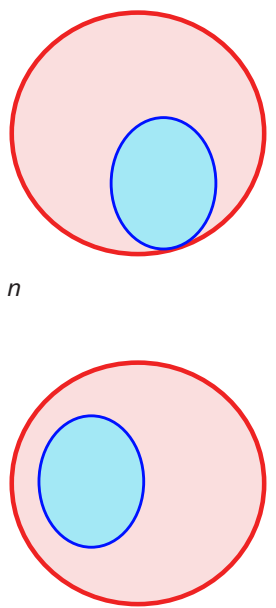

e

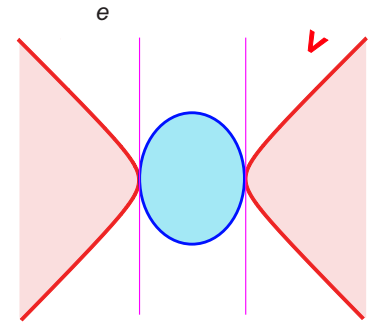

j
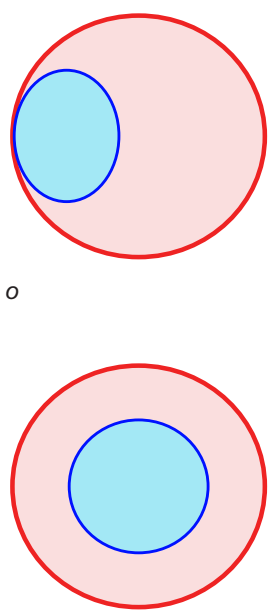

$t$

Figure 1. The conics of the plane $H^{2}$ : a bihyperbola of one sheet in $\widehat{H}(a)$; a bihyperbola of two sheets in $\widehat{H}(b)$; a hyperbolic parabola of one sheet and two branches in $\widehat{H}(c)$; a hyperbolic parabola of two sheets and two branches in $\widehat{H}(d)$; a hyperbolic parabola of one sheet and one branch in $\widehat{H}(\mathrm{e})$; a hyperbola $(f)$; a parabola $(g)$; a hyperbolic cycle of $\widehat{H}(h)$; an equidistance of $\Lambda_{2}(i)$; an elliptic cycle of $\widehat{H}(j)$; a convex elliptic parabole $(k)$; a nonconvex elliptic parabole of $\widehat{H}(1)$; a horocycle of $\widehat{H}(\mathrm{~m})$; an elliptic parabole of $\Lambda_{2}(n)$; a horocycle of $\Lambda_{2}(o)$; a convex ellipce of $\widehat{H}(p)$; a nonconvex ellipce of $\widehat{H}(q)$; a hypercycle of $\widehat{H}(r)$; an ellipce of $\Lambda_{2}(s)$; a circle of $\Lambda_{2}(t)$.

On the plane $H^{2}$ there are seven types of cycles. These types are as follows: the hyperbolic and elliptic cycles of the plane $\widehat{H}$ (see Figures $1 \mathrm{~h}$ and $1 j$, respectively), the equidistantes of the plane $\Lambda_{2}$ (see Figure $1 i$ ), the horocycles of the planes $\widehat{H}$ and $\Lambda_{2}$ (see Figures $1 \mathrm{~m}$ and 10 , respectively), the hypercycles of the plane $\widehat{H}$ (see Figure 1r), the circles of the plane $\Lambda_{2}$ (see Figure $1 t$ ) (see $[4,9,14,11,15]$ ). These conics are paths of an arbitrary point in the plane $H^{2}$ under the transformations of the groop $G$. 


\subsection{Problem statement}

Inversions with respect to the cycles of the plane $H^{2}$ were studied in $[16,18,21]$. Since the absolute of the plane $H^{2}$ can be considered as the limit position of a hypercycle, a horocycle, and a hyperbolic cycle of the plane $\widehat{H}$, as well as of a circle, an equidistant and a horocycle of the plane $\Lambda_{2}$, we enter three types of inversions with respect to the absolute of $H^{2}[19,20]$. An inversion with respect to the absolute of $H^{2}$ we call elliptic, hyperbolic or parabolic if its center is respectively an inner, outer or eigen point with respect to the absolute.

According to the definitions of inverse points, each line containing the inversion center, is invariant under this inversion. We study higher order curves, which are invariant under inversions with respect to the absolute. Such curves we called the Raisa Orbits of the respective order (or $R$-orbits, for short). Depending on the type of the inversion we distinguish elliptic, hyperbolic and parabolic $R$-orbits. Elliptic second-order Raisa Orbits were studied in [20]. In this work we present the hyperbolic second-order $R$-orbits of the plane $H^{2}$. We find canonical equations of these conics and prove that a hyperbolic $R$-orbit of the second order in the plane $H^{2}$ of curvature radius $\rho, \rho \in \mathbb{R}_{+}$, can be a conic of one of four following types:

- the bihyperbolas of one sheet in $\widehat{H}$;

- the hyperbolas;

- the hyperbolic parabolas of one sheet and two branches in $\widehat{H}$;

- the elliptic cycles of radius $\pi \rho / 4$.

In addition, we define the range of each family of all $R$-orbits of the same type.

\subsection{Main notions}

We study the objects of the plane $H^{2}$ in canonical frames of two types. The equations of conics in the frames of the first type allow us to obtain the most informative images by means the interactive mathematics software GeoGebra. The frames of the second type are the most convenient in analytical proofs. Here we briefly provide main notions (see [13, p. 87]). The main definitions and facts on conics in the projective plane are described, for example, in books [1,2].

A canonical frame of the first type of the plane $H^{2}$ is a projective frame $R^{*}=\left\{A_{1}, A_{2}, A_{3}, E\right\}$ whose vertices $A_{1}, A_{2}, A_{3}$ form an autopolar trilateral of the first order with respect to the absolute $\gamma$, and the unit point $E$ lies on the tangents to the curve $\gamma$ drawn from the vertices $A_{1}$ and $A_{2}$.

A canonical frame of the second type of the plane $H^{2}$ is a projective frame $R^{*}=\left\{A_{1}, A_{2}, A_{3}, E\right\}$ whose vertices $A_{1}, A_{2}, A_{3}$ form an autopolar trilateral of the second order with respect to the absolute conic $\gamma$, and points $A_{1}$, $A_{2}$, and $E$ lie on $\gamma$.

The absolute curve $\gamma$ in any canonical frame $R^{*}$ of the first type (or in any canonical frame $R$ of the second type) of the plane $H^{2}$ is given by the equation

$$
x_{1}^{2}+x_{2}^{2}-x_{3}^{2}=0 \quad\left(x_{1} x_{2}-x_{3}^{2}=0\right) .
$$

The tangential equation of the absolute $\gamma$ in any canonical frame $R$ of the second type has the form

$$
4 X_{1} X_{2}-X_{3}^{2}=0
$$

Denote the quadric form $x_{1} x_{2}-x_{3}^{2}$ from the second equation in (1.1) by $\varphi\left(x_{1}, x_{2}, x_{3}\right)$. In the frame $R$, the real coordinates $\left(a_{1}: a_{2}: a_{3}\right)$ of an exterior or interior point with respect to the absolute satisfy respectively the inequality

$$
\varphi\left(a_{1}, a_{2}, a_{3}\right)<0 \quad \text { or } \quad \varphi\left(a_{1}, a_{2}, a_{3}\right)>0 .
$$

Any point of the plane $\widehat{H}$ defines four components in the plane $H^{2}$. Let us determine them by analogy with components of the plane $\widehat{H}$ (see, for instance, [13, p. 104], [14, p. 11], [12, p. 9]).

Let $X$ be an arbitrary point of the plane $\widehat{H}$, and let $l_{1}, l_{2}$ be the parabolic lines containing the point $X$.

By the valiana of a point $X$ in $H^{2}$ we mean the set of all interior points of the angle between lines $l_{1}, l_{2}$ that does not contain the absolute $\gamma$. The polar line $l$ of the point $X$ with respect to $\gamma$ divides the valiana of $X$ into two connected components. We call them the semivalianas of the point $X$.

The complement of the valiana of a point $X$ in $H^{2}$ is called the covaliana of $X$. The covaliana of a point $X$ on $H^{2}$ consists of two components bounded by the lines $l_{1}, l_{2}$, and $l$. We call each of these components the semicovaliana of a point $X$. 


\section{Hyperbolic inversion in the plane $H^{2}$}

Let $\omega(S ; h)$ be a cycle with centre $S$ in the plane $H^{2}$, and let $M$ be an arbitrary point in the plane $H^{2}$. Denote the polar line of the point $M$ with respect to the cycle $\omega$ by $p_{M}$, and the common point of lines $S M$ and $p_{M}$ by $M^{\prime}$.

The points $M$ and $M^{\prime}$ are called inverse points with respect to the cycle $\omega$.

The transformation of the plane $H^{2}$ under which each point $M$ passes to its inverse point $M^{\prime}$ with respect to a cycle $\omega$, is called the inversion with respect to this cycle and is denoted by $I$. The cycle $\omega$ is called the base cycle (or base), the point $S$ is the centre of inversion $I$ (see $[16,18,21]$ ).

When the base cycle $\omega$ is not an elliptic cycle of the plane $\widehat{H}$, the absolute $\gamma$ can be considered as a limit position of $\omega$. When $\omega$ is a hyperbolic cycle of the plane $\widehat{H}$, that is, $S$ is an inner point of the plane $\widehat{H}$, the inversion $I$ is called a hyperbolic inversion with respect to the absolute, or a hyperbolic inversion for short.

Let us find the analytical expression for the hyperbolic inversion $I$ with centre $S$.

To this end we consider a canonical frame $R=\left\{A_{1}, A_{2}, S, E\right\}$ of the second types. For an arbitrary point $M$ of the plane $H^{2}$ with coordinates $\left(m_{1}: m_{2}: m_{3}\right)$ the line $S M$ has the coordinates $\left(-m_{2}: m_{1}: 0\right)$ in $R$. According to the second equation from (1.1) the polar line $p_{M}$ of the point $M$ with respect to the absolute can be set by the coordinates $\left(m_{2}: m_{1}:-2 m_{3}\right)$. Consequently, the point $M^{\prime}$, where $M^{\prime}=I(M)=S M \cap p_{M}$, can be given in $R$ by the coordinates $\left(m_{1} m_{3}: m_{2} m_{3}: m_{1} m_{2}\right)$. Thus, for the inversion $I$ with centre $S$ the following analytical expressions in the frame $R$ hold:

$$
\lambda x_{1}^{\prime}=x_{1} x_{3}, \lambda x_{2}^{\prime}=x_{2} x_{3}, \lambda x_{3}^{\prime}=x_{1} x_{2}, \lambda \in \mathbb{R} .
$$

We formulate and prove several almost obvious assertions in the following theorem.

Theorem 2.1. The hyperbolic inversion I of the plane $H^{2}$ with centre $S$ possesses the following properties.

1. Inversion $I$ is not a bijective transformation of the plane $H^{2}$.

2. Under I a line of the plane $H^{2}$ corresponds to itself if and only if it passes through the point $S$.

3. Let $M, M^{\prime}$ be inverse points under $I$, and let $l$ be the polar line of the point $S$ with respect to the absolute $\gamma$. Then the distance from $M$ to $S$ is equal to the distance from $M^{\prime}$ to $l$.

4. If an arbitrary point $M$ of the plane $H^{2}$ belongs to the covaliana of the point $S$, then the inverse points $M, M^{\prime}$ under I belong to the same semicovaliana of $S$ and to different components $\Lambda_{2}, \widehat{H}$ of the plane $H^{2}$.

5. If an arbitrary point $M$ of the plane $H^{2}$ belongs to the valiana of the point $S$, then the inverse points $M, M^{\prime}$ under $I$ belong to different semivalianas of $S$.

Proof. 1. Accoding to the definition of the hyperbolic inversion each point of a parabolic line containing the point $S$, corresponds under $I$ to the common point of this line and the absolute $\gamma$. Moreover, each point of the polar line of $S$ with respect to $\gamma$ corresponds under $I$ to $S$. This violates the condition of injectiveness of $I$ and thereby proves the first assertion of the theorem.

2. By definition, inverse points under $I$ are collinear with the centre $S$ of $I$. From this follows the second assertion of the theorem.

3. By the condition of the third theorem assertion, the point $S$ is the pole of the line $l$ with resprct to the absolute. Hence, the line $M S$ is orthogonal to the line $l$. Let $M_{0}=M S \cap l$. Then the distance from $M^{\prime}$ to $l$ is equal to $\left|M^{\prime} M_{0}\right|$. Let us prove that $|M S|=\left|M^{\prime} M_{0}\right|$.

Denote the common points of the line $M M^{\prime}$ and the absolute $\gamma$ by $H_{1}, H_{2}$. These points can be real or imaginary conjugate. When the line $M S$ is parabolic, these points coincide. By construct of inverse points, the point $M^{\prime}$ lies on the polar line of $M$ with respect to $\gamma$. Thus, for the cross-ratios $\left(S M_{0} H_{1} H_{2}\right)$, $\left(M M^{\prime} H_{1} H_{2}\right)$ of the quadruples of collinear points the following equalities hold

$$
\left(S M_{0} H_{1} H_{2}\right)=-1, \quad\left(M M^{\prime} H_{1} H_{2}\right)=-1 .
$$

Let us choose a frame $R_{0}=\left\{H_{1}, H_{2}, S\right\}$ on the line $M S$. Accoding to the first equality from (2.2) the point $M_{0}$ has the coordinates $(1:-1)$ in $R_{0}$. Denote coordinates of the points $M, M^{\prime}$ in this frame by $(a: 1)$, 
$(x: 1)$, where $a, x \in \mathbb{R}$. Using the second equality from (2.2), we find that $x=-a$. Expressing the numbers $\left(M S H_{1} H_{2}\right),\left(M^{\prime} M_{0} H_{1} H_{2}\right)$ via coordinates of points in the frame $R_{0}$, we obtain

$$
\left(M S H_{1} H_{2}\right)=\frac{\left|\begin{array}{ll}
a & 1 \\
1 & 0
\end{array}\right|\left|\begin{array}{ll}
1 & 1 \\
0 & 1
\end{array}\right|}{\left|\begin{array}{ll}
a & 1 \\
0 & 1
\end{array}\right|\left|\begin{array}{ll}
1 & 1 \\
1 & 0
\end{array}\right|}=\frac{1}{a}, \quad\left(M^{\prime} M_{0} H_{1} H_{2}\right)=\frac{\left|\begin{array}{cc}
-a & 1 \\
1 & 0
\end{array}\right|\left|\begin{array}{cc}
1 & -1 \\
0 & 1
\end{array}\right|}{\left|\begin{array}{cc}
-a & 1 \\
0 & 1
\end{array}\right|\left|\begin{array}{cc}
1 & -1 \\
1 & 0
\end{array}\right|}=\frac{1}{a} .
$$

Thus, the equality $\left(M S H_{1} H_{2}\right)=\left(M^{\prime} M_{0} H_{1} H_{2}\right)$ holds. Based on this equality and the definition of the length of an elliptic or hyperbolic segment (see, for instance, [13, pp. 118, 122]), we provide support for the equality $|M S|=\left|M^{\prime} M_{0}\right|$, from which the third assertion of the theorem follows.

4. To prove the following assertions, we use the canonical frame $R=\left\{A_{1}, A_{2}, S, E\right\}$ of the second type. By definition, the points $A_{1}, A_{2}, E$ from $R$ lie in $\gamma$, the point $S$ is the pole of the line $A_{1} A_{2}$ with respect $\gamma$.

Let $M$ be an arbitrary point from the covaliana of the point $S$, and let $M_{0}=S M \cap l$, where $l$ is the polar line of $S$ with respect to $\gamma$. Without losing of generality in reasonings we place the unit point $E$ of $R$ on the line $M M^{\prime}$ in the same semicovaliana of $S$ that the point $M$. After that, the points $M, M_{0}$ can be given in the frame $R$ by the coordinates $(1: 1: m), m \in \mathbb{R}$, and $(1: 1: 0)$, respectively. According to formulae (2.1) the point $M^{\prime}$ has the coordinates $(m: m: 1)$. Expressing the cross-ratio $\left(M M^{\prime} M_{0} S\right)$ of the quadruple of collinear points via their coordinates in the frame $R$, we find

$$
\left(M M^{\prime} M_{0} S\right)=\frac{\left|\begin{array}{cc}
1 & m \\
1 & 0
\end{array}\right|\left|\begin{array}{cc}
m & 1 \\
0 & 1
\end{array}\right|}{\left|\begin{array}{cc}
1 & m \\
0 & 1
\end{array}\right|\left|\begin{array}{cc}
m & 1 \\
1 & 0
\end{array}\right|}=m^{2}
$$

Since $\left(M M^{\prime} M_{0} S\right)=m^{2}>0$ for any $m$, the points $M, M^{\prime}$ are not divided by the points $M_{0}, S$. Consequently, $M$ and $M^{\prime}$ lie in the same semicovaliana of the point $S$.

The cross-ratio $\left(M M^{\prime} E S\right)$ has the following expression in coordinates

$$
\left(M M^{\prime} E S\right)=\frac{\left|\begin{array}{cc}
1 & m \\
1 & 1
\end{array}\right|\left|\begin{array}{cc}
m & 1 \\
0 & 1
\end{array}\right|}{\left|\begin{array}{cc}
1 & m \\
0 & 1
\end{array}\right|\left|\begin{array}{cc}
m & 1 \\
1 & 1
\end{array}\right|}=-m
$$

Since the points $M, E$ lie in the same semicovaliana of $S$, the inequality $\left(M E M_{0} S\right)>0$ holds. Writing down this inequality in coordinates, we obtain

$$
\left(M E M_{0} S\right)=\frac{\left|\begin{array}{cc}
1 & m \\
1 & 0
\end{array}\right|\left|\begin{array}{cc}
1 & 1 \\
0 & 1
\end{array}\right|}{\left|\begin{array}{cc}
1 & m \\
0 & 1
\end{array}\right|\left|\begin{array}{cc}
1 & 1 \\
1 & 0
\end{array}\right|}=m>0
$$

By the conditions (2.3), (2.4), the inequality $\left(M M^{\prime} E S\right)<0$ holds. Consequently, the points $M, M^{\prime}$ are divided by the points $E, S$, that is, the points $M, M^{\prime}$ belong to different components $\Lambda_{2}, \widehat{H}$ of the plane $H^{2}$. So, the fourth assertion of the theorem is proved.

5. Now we assume that an arbitrary point $M$ lies in the valiana of the point $S$. Keeping the previous notations, we place the unit point $E$ of the frame $R$ on the line, containing the point $S$ and orthogonal to the line $S M$. The points $M, M_{0}$ have the coordinates $(1:-1: m), m \in \mathbb{R}$, and $(1:-1: 0)$, respectively. By formulae (2.1), the point $M^{\prime}$ has the coordinates $(-m: m: 1)$. Hence, for the cross-ratio $\left(M M^{\prime} M_{0} S\right)$ we obtain

$$
\left(M M^{\prime} M_{0} S\right)=\frac{\left|\begin{array}{cc||cc}
1 & m \\
1 & 0
\end{array}\right|\left|\begin{array}{cc}
-m & 1 \\
0 & 1
\end{array}\right|}{\left|\begin{array}{cc}
1 & m \\
0 & 1
\end{array}\right|\left|\begin{array}{cc}
-m & 1 \\
1 & 0
\end{array}\right|}=-m^{2} .
$$


Since $\left(M M^{\prime} M_{0} S\right)=-m^{2}<0$ for any $m$, the points $M, M^{\prime}$ are divided by the points $M_{0}, S$. Consequently, the points $M, M^{\prime}$ belong to different semivaliana of the point $S$. This completes the theorem proof.

\section{Main Theorem on hyperbolic Raisa Orbits of the second order}

Theorem 3.1. The family $\Theta$ of all hyperbolic Raisa Orbits of the second order in the plane $H^{2}$ of curvature radius $\rho$, $\rho \in \mathbb{R}_{+}$, possesses the following properties.

1. Each conic from $\Theta$ belongs to one of four conics types of the plane $H^{2}$. These types are as follows: the bihyperbolas of one sheet in $\widehat{H}$; the hyperbolas; the hyperbolic parabolas of one sheet and two branches in $\widehat{H}$; the elliptic cycles of radius $\pi \rho / 4$.

2. The family of all bihyperbolas of one sheet in $\widehat{H}$ (or all hyperbolas) defined exactly up to motions and belonging to the family $\Theta$, is one-parametric. Each conic from this family can be set in some canonical frame of the second type by the equation

$$
x_{3}^{2}+x_{1} x_{2}+a x_{1} x_{3}-(a+2) x_{2} x_{3}=0, a \in \mathbb{R}, a \neq-1 \quad\left(x_{3}^{2}+x_{1} x_{2}+a\left(x_{1} x_{3}+x_{2} x_{3}\right)=0, a \in \mathbb{R},|a|>1\right) .
$$

All hyperbolic R-orbits from the hyperbolas family belong to the same class of hyperbolas.

3. The family $\Theta$ contains a unique hyperbolic parabola of one sheet and two branches in $\widehat{H}$ and a unique elliptic cycle, which are defined exactly up to motions. A hyperbolic parabola of one sheet and two branches in $\widehat{H}$ (or elliptic cycle) from $\Theta$ can be set in some canonical frame of the second type by the equation

$$
x_{3}^{2}+x_{1} x_{2}-2 x_{1} x_{3}=0, \quad\left(x_{1} x_{2}+x_{3}^{2}=0\right) .
$$

Proof. Let non-degenerate conic $\sigma$ of the plane $H^{2}$ be invariant under hyperbolic inversion $I$ with centre $S$. To find all posible types of $\sigma$ as an oval curve of the plane $H^{2}$, we choose a canonical frame $R=\left\{A_{1}, A_{2}, S, E\right\}$ of the second type. The family $U$ of all such frames depends on one parameter. Indeed, the point $S$ is fixed. Hence, its polar line $A_{1} A_{2}$ with respect to the absolute $\gamma$ is fixed too. Moreover, by the frame definition, $A_{1} \in \gamma, A_{2} \in \gamma$. Thus, the vertices $A_{1}$ and $A_{2}$ of the frame $R$ are defined with exactly up the points order. The unit point $E$ can move along $\gamma$, that is, its position depends on one parameter. This parameter defines a frame in the family $U$.

If $S$ lies in $\sigma$, then each line containing the point $S$, except for the tangent of the conic $\sigma$, has another point from $\sigma$. Assume that a line $t$ contains $S$ and $t \cap \sigma=\{S, V\}$, where $V \neq S$. The point $V^{\prime}$, where $V^{\prime}=I(V)$, lies in both the line $t$ and the conic $\sigma$. Hence $t$ contains three points from the conic $\sigma$. This is not possible because the conic $\sigma$ is non-degenerate. Thus, $\sigma$ does not contain the point $S(0: 0: 1)$. In that case, the conic $\sigma$ can be given in the frame $R$ by the equation

$$
\sum_{i, j=1}^{3} a_{i j} x_{i} x_{i}=0, a_{i j} \in \mathbb{R}, a_{33}=1 .
$$

Using the analytical expression (2.1) of the inversion $I$, we get the equation of a curve $\sigma^{\prime}$, containing image of the conic $\sigma$ under $I$

$$
a_{11} x_{1}^{2} x_{3}^{2}+a_{22} x_{2}^{2} x_{3}^{2}+x_{1}^{2} x_{2}^{2}+2 a_{12} x_{1} x_{2} x_{3}^{2}+2 a_{13} x_{1}^{2} x_{2} x_{3}+2 a_{23} x_{1} x_{2}^{2} x_{3}=0 .
$$

Since $\sigma$ is invariant under $I$, the curve $\sigma^{\prime}$ breaks down into a pair of conics, one of which is $\sigma$. Let $\sigma^{\prime}=\sigma \cup \beta$, where the conic $\beta$ is given in $R$ by the equation

$$
\sum_{i, j=1}^{3} b_{i j} x_{i} x_{i}=0, b_{i j} \in \mathbb{R} .
$$

Comparing the product of quadric forms from Eqs. (3.3), (3.5) with the quadric form from Eq. (3.4) under the condition $a_{33}=1$, we obtain the following conditions for coefficients $a_{i j}$ and $b_{i j}$ :

$$
a_{11}=a_{22}=0,2 a_{12}=1, b_{11}=b_{22}=b_{33}=b_{13}=b_{23}=0, b_{12} \neq 0 .
$$

Under these conditions the conic $\beta$ has the equation $x_{1} x_{2}=0$ and is the pair of parabolic lines containing $S$. The conic $\sigma$ equation can be written in the form

$$
x_{3}^{2}+x_{1} x_{2}+a x_{1} x_{3}+b x_{2} x_{3}=0, \quad a, b \in \mathbb{R}, \quad a b \neq 1 .
$$


Denote the quadric form $x_{3}^{2}+x_{1} x_{2}+a x_{1} x_{3}+b x_{2} x_{3}$ from Eq. (3.6) by $\varphi_{0}\left(x_{1}, x_{2}, x_{3}\right)$. The determinant of a coordinate matrix of the form $\varphi_{0}\left(x_{1}, x_{2}, x_{3}\right)$ is equal to $(a b-1) / 4$. Hence the condition $a b \neq 1$ guarantees the nondegeneracy of the conic $\sigma$.

We investigate the hyperbolic $R$-orbit $\sigma$, considering all fundamentally different possibilities for coefficients $a, b$ from Eq. (3.6).

1. At first, we assume that the conic $\sigma$ is given by Eq. (3.6), where $a b \neq 0$. Under this condition from Eqs. (1.1), (3.6) we obtain coordinates of common points of the $R$-orbit $\sigma$ and the absolute $\gamma$ in $R$ :

$$
\begin{aligned}
& A_{1}(1: 0: 0), Q_{1}(b(1+\sqrt{1-a b}): a(1-\sqrt{1-a b}):-a b), \\
& A_{2}(0: 1: 0), Q_{2}(b(1-\sqrt{1-a b}): a(1+\sqrt{1-a b}):-a b) .
\end{aligned}
$$

Note that the points $A_{1}, A_{2}$ are real, and under the conditions $a b \neq 1, a b \neq 0$ there are no matching points in the set $A_{1}, A_{2}, Q_{1}, Q_{2}$. According to the nature of the points $Q_{1}, Q_{2}$ we distinguish the following cases.

(a) Assume that $a b<1$. In this case the points $Q_{1}, Q_{2}$ are real. Consequently, $\sigma$ has four real points from the absolute. By the classification of oval curves of the plane $\widehat{H}$ (see $[14,11])$, the conic $\sigma$ can be a bihyperbola of one sheet or a bihyperbola of two sheets in $\widehat{H}$ (see Figures $1 \mathrm{a}$ and $1 b$, respectively). Among the diagonal points of a full quadrilateral with vertices at the common points of the absolute and a bihyperbola of two sheets, there is a point which is interior both with respect to the absolute and with respect to the bihyperbola. We use this fact to clarify the conic $\sigma$ type. Let us consider the full quadrilateral $A_{1} A_{2} Q_{1} Q_{2}$. Let $A_{1} A_{2} \cap Q_{1} Q_{2}=A, A_{1} Q_{1} \cap A_{2} Q_{2}=B$, and $A_{1} Q_{2} \cap A_{2} Q_{1}=C$. The diagonal points $A, B, C$ of the full quadrilateral $A_{1} A_{2} Q_{1} Q_{2}$ have the following coordinates in $R$ :

$$
\begin{gathered}
A(b:-a: 0), \quad B(b(1-\sqrt{1-a b}): a(1-\sqrt{1-a b}):-a b), \\
C(b(1+\sqrt{1-a b}): a(1+\sqrt{1-a b}):-a b) .
\end{gathered}
$$

Let $K$ be a pole of the line $A_{1} A_{2}$ with respect $\sigma$. Since the points $A_{1}, A_{2}$ are real, the point $K$ is an exterior point with respect $\sigma$. Using coordinates $(b: a:-1)$ of the point $K$, we find the following inequalities for real coordinates $\left(a_{1}: a_{2}: a_{3}\right)$ of an arbitrary exterior (interior) point with respect to the conic $\sigma$ in the frame $R$ under the condition $a b<1$ :

$$
\varphi_{0}\left(a_{1}, a_{2}, a_{3}\right)>0 \quad\left(\varphi_{0}\left(a_{1}, a_{2}, a_{3}\right)<0\right) .
$$

The quadric forms $\varphi$ and $\varphi_{0}$ on the coordinates of points $A, B, C$ have the following expressions:

$$
\begin{gathered}
\varphi(A)=-a b, \quad \varphi_{0}(A)=-a b, \\
\varphi(B)=-2 a b \sqrt{1-a b}(1-\sqrt{1-a b}), \quad \varphi_{0}(B)=2 a b(1-a b)(1-\sqrt{1-a b}), \\
\varphi(C)=2 a b \sqrt{1-a b}(1+\sqrt{1-a b}), \quad \varphi_{0}(C)=2 a b(1-a b)(1+\sqrt{1-a b}) .
\end{gathered}
$$

Using these expressions under the condition $a b<1$, we proved the following inequalities:

$$
\varphi(A) \varphi_{0}(A)>0, \quad \varphi(B)<0, \quad \varphi_{0}(B)>0, \quad \varphi(C) \varphi_{0}(C)>0 .
$$

By virtue of the inequalities (1.3), (3.7), and (3.8), the following assertions hold:

1 ) the point $A$ (or $C$ ) is interior with respect to the conic $\sigma$ if and only if it is exterior with respect to the absolute;

2) the point $B$ is exterior with respect to the conic $\sigma$ and with respect to the absolute.

Thus, none of the points $A, B, C$ lie in the intersection of the interior domains of the conics $\gamma$ and $\sigma$. From this we conclude that under the conditions $a b \neq 0, a b<1$ the conic $\sigma$ is a bihyperbola of one sheet in the plane $\widehat{H}$.

Note that Eq. (3.6) contains two parameters $a, b$, while the point $E$ position depends on one parameter. By fixing the point $E$, for example, at the point $Q_{1}$, we obtain the condition $b=-a-2$, that is, we will reduce the number of parameters in Eq. (3.6) to one. After that, the frame $R$ is determined uniquely, and the conic $\sigma$ can be set in $R$ by the first equation from (3.1). 
(b) Assume that $a b>1$. In this case the points $Q_{1}, Q_{2}$ are imaginary conjugate. Consequently, the conic $\sigma$ has two real and two imaginary points from the absolute. By the classification of oval curves of the plane $\widehat{H}$, this means that the conic $\sigma$ is a hyperbole (see Figure 1f). The class of a hyperbola in the plane $\widehat{H}$ is defined by the position of poles of the line connecting the common real points of the hyperbola and the absolute with respect to the absolute and with respect to the hyperbola. There are nine classes of hyperbolas of the plane $\widehat{H}$ (see [11, p. 44]).

Under the condition $a b>1$ real coordinates $\left(a_{1}: a_{2}: a_{3}\right)$ of an arbitrary interior point with respect to the conic $\sigma$ satisfy the inequality $\varphi_{0}\left(a_{1}, a_{2}, a_{3}\right)>0$. Hence the point $S(0: 0: 1)$ lies in the interior domain with respect $\sigma$. By virtue of the inequalities (1.3) and $a b>1$, the point $K(b: a:-1)$ lies in the interior domain with respect $\gamma$. Therefore, all hyperbolic $R$-orbits from the hyperbolas family belong to the same class of hyperbolas.

Note that Eq. (3.6) contains two parameters $a, b$ under such condition that the point $E$ position depends on one parameter. By fixing the point $E$, for example, in the line $S K$, we obtain the condition $a=b$, that is, we will reduce the number of parameters in Eq. (3.6) to one. After that, the frame $R$ is determined uniquely, and the conic $\sigma$ can be set in $R$ by the second equation from (3.1).

2. Now we assume that the conic $\sigma$ is given by Eq. (3.6), where $a \neq 0, b=0$. The case, when $a=0, b \neq 0$, gives a similar result because it can be obtained by permuting vertices $A_{1}, A_{2}$ of the frame $R$.

From Eqs. (1.1), (3.6) we find coordinates of common points of the $R$-orbit $\sigma$ and the absolute:

$$
A_{1}(1: 0: 0), \quad A_{2}(0: 1: 0), \quad Q\left(4: a^{2}:-2 a\right), a \neq 0 .
$$

Since there are no matching points in the set $A_{1}, A_{2}, Q$, the conic $\sigma$ is a hyperbolic parabola (see [14, p. 29]). Each hyperbolic parabola of the plane $H^{2}$ belongs to one of three types (see Figures $1 c, 1 d$, and 1 e). We refine the conic $\sigma$ type, taking into account the number and nature of its common tangents with the absolute.

In the case on consideration the tangential equation of the conic $\sigma$ can be written in the form

$$
a^{2} X_{2}^{2}+X_{3}^{2}+4 X_{1} X_{2}-2 a X_{2} X_{3}=0, \quad a \neq 0 .
$$

From the system of Eqs. (1.2), (3.9) we find common tangents of the conics $\sigma$ and $\gamma$ :

$$
A_{2} A_{3}(1: 0: 0), \quad l_{1}^{*}\left(a^{2}: 8 i: 4 a(1+i)\right), \quad l_{2}^{*}\left(a^{2}:-8 i: 4 a(1-i)\right) .
$$

Two of three common tangents of the conics $\sigma$ and $\gamma$ are imaginary. Hence the conic $\sigma$ is a hyperbolic parabola of one sheet and two branches in $\widehat{H}$.

By fixing the point $E$ at the point $Q$, we obtain the condition $a=-2$, that is, we fix the last free parameter $a$ in Eq. (3.6) under $b=0$. In this step, the frame $R$ is fixed, and the conic $\sigma$ can be set in $R$ by the first equation from (3.2).

3. Finally, we assume that the conic $\sigma$ is given by Eq. (3.6), where $a=b=0$. In this case, the conic $\sigma$ is not depend on the point $E$ position and can be set by the second equation from (3.2). It is an elliptic cycle of the plane $H^{2}$ with centre $S$ and radius $\pi \rho / 4$ (see [14, p. 39]).

We considered all possible cases and uncovered four types of hyperbolic second-order $R$-orbits in the plane $H^{2}$. This yields the first theorem assertion. In addition, for each conic under study we presented the canonical frame in which the conic can be given by one of the equations from (3.1), (3.2). In each case, having regard to the number of free parameters of the equations from (3.1), (3.2), we get the second and third assertions of the theorem. Thus, the theorem is proved.

\section{Visualization of stadied objects}

In order to display the studied conics, we represent them in the plane $E_{2}$ (see, for instance, $[4,17,22]$ ). 
Let the coordinate line $A_{1} A_{2}$ of the frame $R$ be the absolute line of the plane $E_{2}$. The absolute circle-points $J_{1}$ and $J_{2}$ of $E_{2}$ we give by coordinates $( \pm i: 1: 0)$. Under this agreement the following relations between the Cartesian coordinates $(x, y)$ and the projective coordinates $\left(x_{1}: x_{2}: x_{3}\right)$ of the same point hold

$$
x=\frac{x_{1}}{x_{3}}, \quad y=\frac{x_{2}}{x_{3}} .
$$

Using the relations (4.1), we move from Eqs. (1.1), (3.1), and (3.2) to the following equations of the absolute $\gamma$ and hyperbolic $R$-orbits in the Cartesian coordinates $(x, y)$ corresponding to the frame $R$ :

$$
\begin{gathered}
\gamma: x y-1=0, \\
x y+a x-(a+2) y+1=0, a \in \mathbb{R}, a \neq-1, \\
x y+a(x+y)+1=0, a \in \mathbb{R},|a|>1, \\
x y-2 x+1=0, \\
x y+1=0 .
\end{gathered}
$$

We construct images of the absolute and hyperbolic second-order $R$-orbits in the plane $E_{2}$ through the equations (4.2), (4.3), (4.4), (4.5), (4.6) in the Cartesian system corresponding to the frame $R$. The obtained conics are shown in Figures 1a, 2a, 3a, and $4 a$. The interior domains with respect to the conics $\gamma$ and $\sigma$ are highlighted.

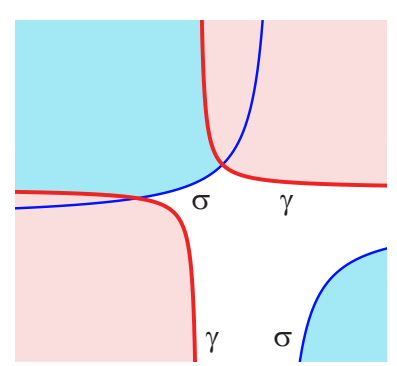

a

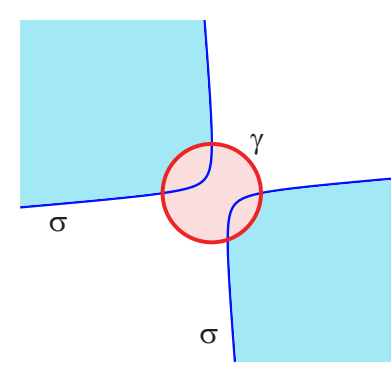

Figure 2. Images of the absolute $\gamma$ of the plane $H^{2}$ and the hyperbolic second-order $R$-orbit $\sigma$ with Eqs. (4.3) (a), (4.14) $(b)$, where $a=5$, in $E_{2}$. The conic $\sigma$ is a bihyperbola of one sheet.

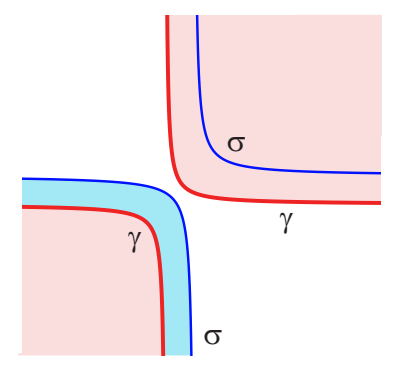

a

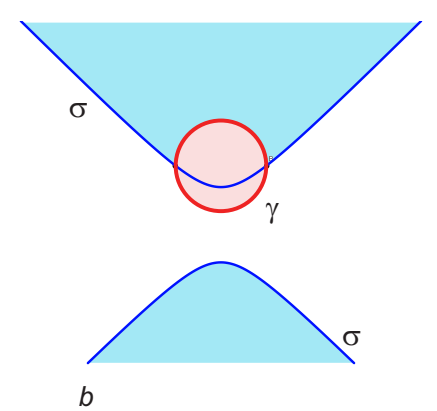

Figure 3. Images of the absolute $\gamma$ of the plane $H^{2}$ and the hyperbolic second-order $R$-orbit $\sigma$ with Eqs. (4.4) (a), (4.15) (b), where $a=1.3$, in $E_{2}$. The conic $\sigma$ is a hyperbola.

To get the most informative images of the studied objects on the Euclidean plane $E_{2}$, we will go to the canonical frame $R^{*}$ of the first type. Let us obtain the formulae for transforming the coordinates of an arbitrary point in the plane $H^{2}$ which correspond to this transition.

Assume that the canonical frame $R^{*}=\left\{A_{1}^{*}, A_{2}^{*}, A_{3}^{*}, E^{*}\right)$ of the first type associated with the canonical frame $R=\left\{A_{1}, A_{2}, A_{3}, E\right)$ of the second type by the following conditions:

$$
A_{1}^{*}=E_{21}=A_{1}-A_{2}, \quad A_{2}^{*}=A_{3}, \quad A_{3}^{*}=E_{12}=A_{1}+A_{2}, \quad E^{*}=A_{2} A_{3} \cap E E_{21} .
$$




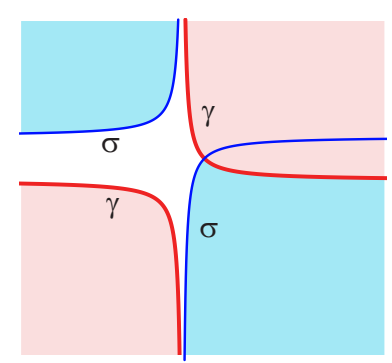

a

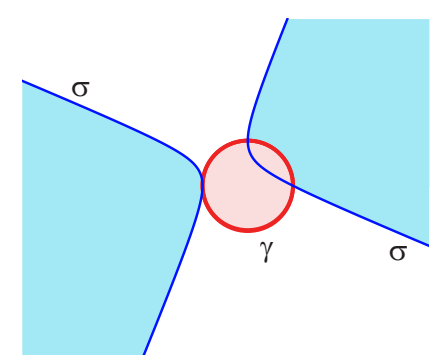

$b$

Figure 4. Images of the absolute $\gamma$ of the plane $H^{2}$ and the hyperbolic second-order $R$-orbit $\sigma$ with Eqs. (4.5) (a), (4.16) (b) in $E_{2}$. The conic $\sigma$ is a hyperbolic parabola of one sheet and two branches.

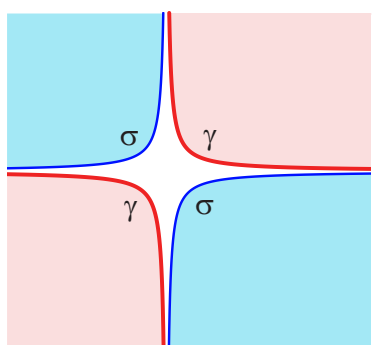

$a$

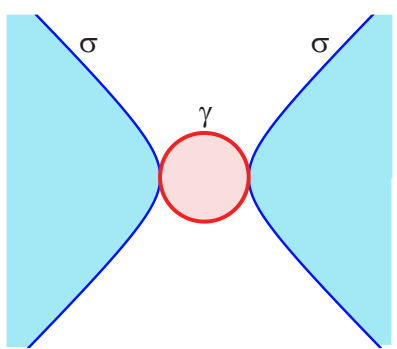

$b$

Figure 5. Images of the absolute $\gamma$ of the plane $H^{2}$ and the hyperbolic second-order $R$-orbit $\sigma$ with Eqs. (4.6) (a), (4.17) (b) in $E_{2}$. The conic $\sigma$ is an elliptic cycle.

Under these conditions the vertices and unit point of the frame $R^{*}$ have the following coordinates in the frame $R$ :

$$
A_{1}^{*}(1:-1: 0), \quad A_{2}^{*}(0: 0: 1), \quad A_{3}^{*}(1: 1: 0), \quad E^{*}(2: 0: 1) .
$$

Consequently, the compatible matrix of the transition from $R$ to $R^{*}$ can be written in the form:

$$
\left(\begin{array}{cccc}
1 & 0 & 1 & 2 \\
-1 & 0 & 1 & 0 \\
0 & 1 & 0 & 1
\end{array}\right) .
$$

Denote the coordinates of an arbitrary point of the plane $H^{2}$ in the frame $R$ by $\left(x_{1}: x_{2}: x_{3}\right)$ and denote the coordinates of the same point in the frame $R^{*}$ by $\left(x_{1}^{*}: x_{2}^{*}: x_{3}^{*}\right)$. Using the matrix (4.7), we get the desired formulae of the coordinates transformation:

$$
\lambda x_{1}=x_{1}^{*}+x_{3}^{*}, \quad \lambda x_{2}=-x_{1}^{*}+x_{3}^{*}, \quad \lambda x_{3}=x_{2}^{*}, \quad \lambda \in \mathbb{R} .
$$

Rewriting the equations of investigated conics from (3.1), (3.2) using the formulae (4.8), we obtain the equations of these conics in the frame $R^{*}$ :

$$
\begin{gathered}
-x_{1}^{2}+x_{2}^{2}+x_{3}^{2}+2(a+1) x_{1} x_{2}-2 x_{2} x_{3}=0, a \in \mathbb{R}, a \neq-1, \\
-x_{1}^{2}+x_{2}^{2}+x_{3}^{2}+2 a x_{2} x_{3}=0, a \in \mathbb{R},|a|>1, \\
-x_{1}^{2}+x_{2}^{2}+x_{3}^{2}-2 x_{1} x_{2}-2 x_{2} x_{3}=0 \\
-x_{1}^{2}+x_{2}^{2}+x_{3}^{2}=0 .
\end{gathered}
$$

Using the relations (4.1), we move from Eqs. (1.1), (4.9) - (4.12) to the following equations of the absolute $\gamma$ and hyperbolic $R$-orbits in the Cartesian coordinates $(x, y)$ corresponding to the frame $R^{*}$ :

$$
\begin{gathered}
\gamma: x^{2}+y^{2}=1 . \\
x^{2}-y^{2}-2(a+1) x y+2 y=1, a \in \mathbb{R}, a \neq-1, \\
x^{2}-y^{2}-2 a y=1, a \in \mathbb{R},|a|>1,
\end{gathered}
$$




$$
\begin{gathered}
x^{2}-y^{2}+2 x y+2 y=1, \\
x^{2}-y^{2}=1 .
\end{gathered}
$$

By applying the equations (4.13), (4.14), (4.15), (4.16), (4.17), we construct images of the absolute and hyperbolic second-order $R$-orbits in the Cartesian system corresponding to the frame $R^{*}$. The obtained conics are shown in Figures $1 b, 2 b, 3 b$, and $4 b$.

\section{References}

[1] Busemann, H., Kelly P.: Projective Geometry and Projective Metrics. Academic Press Inc. New York (1953).

[2] Glaeser G., Stachel H., Odehnal B.: The Universe of conics. From the ancient Greeks to 21st century developments. Springer Spektrum (2016).

[3] Halbeisen L., Hungerbühler N.: The exponential pencil of conics. Beitr. Algebra Geom., 59, 549-571 (2018).

[4] Klein, F.: Vorlesungen über Nicht-Euclidische Geometrie. Verlag von Julius Springer. Berlin (1928).

[5] Liebmann, H.: Nichteuklidische geometrie. Leipzig (1912).

[6] Petitjean, S.: Invariant-based characterization of the relative position of two projective conics. In: Emiris I., Sottile F., Theobald T. (eds) Nonlinear Computational Geometry. The IMA Volumes in Mathematics and its Applications. Springer. New York. 151. 189-220 (2009).

[7] Pevzner, S.: Properties of second-order curves in the Lobatchevsky plane which are dual under the focal directrix. Izv. Vyssh. Uchebn. Zaved. Mat. 5, 39-50 (1961).

[8] Pevzner, S.: A detailed classification of second-order irreducible curves in the Lobatchevsky plane by means of the focal-director invariants. Izv. Vyssh. Uchebn. Zaved. Mat. 6, 85-90 (1962).

[9] Rosenfel'd, B.: Noneuclidean spaces. Nauka. Moscow (1969).

[10] Rosenfel'd, B., Zamakhovskii M.: Geometry of Lie groups. Symmetric, parabolic and periodic spaces. Moscow center for countinuous mathematical education. Moscow (2003).

[11] Romakina, L.: Oval lines of the hyperbolic plane of positive curvature. Izv. Sarat. Univ. (N. S.), Ser. Mat. Mekh. Inform. 12 (3), 37-44 (2012).

[12] Romakina, L.: Simple partitions of a hyperbolic plane of positive curvature. Sbornik: Mathematics, 203 (9), 1310-1341 (2012). Translated from Matematicheskii Sbornik 203 (9), 83-116 (2012).

[13] Romakina, L.: Geometry of the hyperbolic plane of positive curvature. P. 1: Trigonometry. Publishing house of the Saratov university. Saratov (2013).

[14] Romakina, L.: Geometry of the hyperbolic plane of positive curvature. P. 2: Transformations and simple partitions. Publishing house of the Saratov university. Saratov (2013).

[15] Romakina, L.: Cycles on the hyperbolic plane of positive curvature. J. Mat. Sciences. 212 (5), 605-621 (2016). Translated from Zap. Nauchn. Sem. S.-Peterburg. Otdel. Mat. Inst. Steklov. (POMI) 415, 137-162 (2013).

[16] Romakina, L.: Inversion with respect to a hypercycle of a hyperbolic plane of positive curvature. Journal of Geometry. 107 (1), 137-149 (2016).

[17] Romakina, L.: Svetlana Ribbons with intersecting axes in a hyperbolic plane of positive curvature. Journal for Geometry and Graphics, 20 (2), 209-224 (2016).

[18] Romakina, L.: Inversion with respect to a horocycle of a hyperbolic plane of positive curvature. Tr. Inst. Mat. 25 (2), 82-92 (2017).

[19] Romakina, L.: Inversion with respect to the absolute of an extended hyperbolic plane. In: Proceedings of the International Forum of Mathematical Education dedicated to the 225th anniversary of N.I. Lobachevskii, Oct 18-22/2017, Kazan, RUSSIA. Publishing House of Kazan University. 111-114 (2017).

[20] Romakina, L.: Elliptic R-orbits of the second order in an extended hyperbolic plane. In: Effective researches of modernity, Scientific articles collection of the 10th Internat. Scientific Conference of Eurasian Scientific Association, Moscow, Oct. 2018, 10, 18-21 (2018).

[21] Romakina, L.: Inversion with respect to an elliptic cycle of a hyperbolic plane of positive curvature. Tr. Inst. Mat. 27 (1-2), 60-78 (2019).

[22] Romakina, L.: Construction of cubic curves with a node. Beitr. Algebra Geom., 60 (4), 761-781 (2019).

\section{Affiliations}

LYUDMILA N. ROMAKINA

ADDRESS: Saratov State University, Dept. of Geometry, Saratov, Russia.

E-MAIL: romakinaln@mail.ru

ORCID ID: 0000-0002-3695-2076 